\title{
Is it possible to predict the onset of Graves' disease?
}

\author{
Nobuyuki Amino
}

Ideally, medicine enables us to prevent disease; failing that, it should allow us to detect and diagnose diseases at an early stage, and to stop them from progressing to a severe state. Endocrine diseases are no exception to these ideals. Autoimmune thyroid disease is a frequently observed illness (predominantly affecting females), and hyperthyroid or hypothyroid conditions markedly disturb the quality of life. It is well established that hyperthyroidism in Graves' disease is induced by antibodies against the TSH receptor (TRAb) that stimulate the thyroid gland. Sensitive assay techniques for the detection of TRAb have been developed, and these show a positive reaction in $99 \%$ of patients with Graves' disease. In other autoimmune diseases, detection of the appropriate autoantibodies often predicts the clinical onset of disease. Detection of TRAb before disease onset might similarly enable us to predict which patients are likely to develop Graves' disease and, therefore, allow intervention to prevent disease onset.

Maternal thyroid dysfunction is commonly observed after delivery because of postpartum aggravation of subclinical autoimmune thyroiditis, which exists in about $10 \%$ of adult females. During pregnancy, immune reactions are suppressed to avoid rejection of the fetus (which has paternal antigens). After delivery, however, immune reactions are enhanced compared with before pregnancy because of the immune rebound mechanism; as a result, 5-10\% of women develop various types of thyroid dysfunction postpartum. Around 5\% of these women develop postpartum Graves' disease. In New York, it was reported that in parous women with Graves' disease, $45 \%$ were diagnosed in the postpartum period (Benhaim Rochester D and Davies TF [2005] Thyroid 15: 1287-1290). Hidaka Y et al. ([1994] Clin Endocrinol 41: 15-20) examined 3,405 consecutive women early in pregnancy and found that $7.7 \%$ of them had antibodies against thyroid

\section{It might be possible to predict the postpartum onset of Graves" disease, if a sensitive enough assay is used in early pregnancy}

NAmino is a consultant physician at Kuma Hospital, Center for Excellence in Thyroid Care, Kobe 650-0011, Japan.

\section{Competing interests}

The author declared he has no competing interests.

www.nature.com/clinicalpractice doi:10.1038/ncpendmet0326 microsomes. Of the 71 of these antibodypositive women examined after pregnancy, 5 developed Graves' disease. Hidaka et al. confirmed that all the women with postpartum Graves' disease had TRAb in early pregnancy that were detectable by highly sensitive bioassay. It might be possible, therefore, to predict the postpartum onset of Graves' disease, if a sensitive enough assay for TRAb is used in early pregnancy; however, cost-effectiveness of this approach should be considered.

Predicted cases of postpartum Graves' disease could, moreover, be prevented by short-term corticosteroid therapy initiated immediately after delivery, similar to the prevention of postpartum development of hypothyroidism (Tada $\mathrm{H}$ et al. [2003] Int J Endocrinol Metab 2: 48-54). Postpartum Graves' disease is estimated to account for around $20 \%$ of all cases of Graves' disease (including those affecting males). Postpartum prevention could, therefore, eliminate a significant proportion of Graves' disease, and serve as a good model of prevention of postpartum onset of other autoimmune diseases.

This procedure to predict disease could also apply to the prevention of Graves' disease not related to pregnancy. In the subjects with family history of autoimmune thyroid disease, or other autoimmune diseases and/or goiter, screening for autoantibodies against thyroid microsomes or peroxidase might be useful for detection of subclinical autoimmune thyroid abnormalities. If subjects show positive reaction, a second screening for TRAb might be useful to detect the preonset of Graves' disease. We should pay particular attention to subjects who have allergic rhinitis or those who are planning to have therapy with a gonadotropin-releasing-hormone analog, since these factors can induce the onset of Graves' disease (Hidaka Y et al. [1996] Thyroid 6: 349-351; Amino N et al. [2003] Thyroid 13: 815-818). 